\title{
On Thermal-Pulse-Driven Plasma Flows in Coronal Funnels as Observed by the Hinode/EUV Imaging Spectrometer (EIS)
}

\author{
A.K. Srivastava $\cdot$ P. Konkol $\cdot$ K. Murawski · \\ B.N. Dwivedi · A. Mohan
}

Received: 23 January 2014 / Accepted: 28 July 2014 / Published online: 21 August 2014

(C) The Author(s) 2014. This article is published with open access at Springerlink.com

\begin{abstract}
Using one-arcsecond-slit-scan observations from the Hinode/EUV Imaging Spectrometer (EIS) on 5 February 2007, we find the plasma outflows in the open and expanding coronal funnels at the eastern boundary of AR 10940. The Doppler-velocity map of Fe XII $195.120 \AA$ shows the diffuse closed-loop system to be mostly red-shifted. The open arches (funnels) at the eastern boundary of AR exhibit blue-shifts with a maximum speed of about $10-15 \mathrm{~km} \mathrm{~s}^{-1}$. This implies outflowing plasma through these magnetic structures. In support of these observations, we perform a 2D numerical simulation of the expanding coronal funnels by solving the set of ideal MHD equations in appropriate VAL-III C initial temperature conditions using the FLASH code. We implement a rarefied and hotter region at the footpoint of the model funnel, which results in the evolution of slow plasma perturbations propagating outward in the form of plasma flows. We conclude that the heating, which may result from magnetic reconnection, can trigger the observed plasma outflows in such coronal funnels. This can transport mass into the higher corona, giving rise to the formation of the nascent solar wind.
\end{abstract}

Keywords MHD · Magnetic fields · Corona · Waves

Electronic supplementary material The online version of this article

(doi:10.1007/s11207-014-0584-9) contains supplementary material, which is available to authorized users.

A.K. Srivastava · B.N. Dwivedi · A. Mohan

Department of Physics, Indian Institute of Technology (Banaras Hindu University), Varanasi 221005, India

A.K. Srivastava

e-mail: asrivastava.app@iitbhu.ac.in

P. Konkol · K. Murawski (凶)

Group of Astrophysics, UMCS, ul. Radziszewskiego 10, Lublin 20-031, Poland

e-mail: kmurawski@umcs.lublin.pl 


\section{Introduction}

The solar wind is the supersonic outflow of fully ionized gas from the solar corona streaming along the magnetic-field lines. It is well established that the polar coronal hole is the source of fast solar wind (Hassler et al., 1999; Wilhelm et al., 2000; Tu et al., 2005), while the slow solar wind originates from the boundary of active regions and along the streamers in the equatorial corona (Habbal et al., 1997; Sakao, Kano, and Narukage, 2007). Hassler et al. (1999) have found that the plasma can be supplied from the chromospheric heights in the network boundaries to the solar wind in polar coronal holes. More precise estimation of the formation of nascent wind in coronal funnels between 5-20 Mm in coronal holes has been carried out by Tu et al. (2005). The bases of the polar coronal holes are mostly the sources of fast solar wind. It has also been suggested that plasma outflows observed at the edges of active regions are the source of the slow solar wind. Active-region arches, which can extend outward as the rays in the outer corona, may channel it (Slemzin et al., 2013). In addition to the large-scale origin, it has also been found that the small-scale outflows at the coronal hole boundaries (CHBs) can serve as the source of the slow solar wind (Subramanian, Madjarska, and Doyle, 2010). Recently, Yang et al. (2013) have presented a numerical model to describe the process of magnetic reconnection between moving magnetic features (MMFs) and the pre-existing ambient magnetic field that drives an anemone jet with inverted $y$-shape base and associated plasma blobs. They have found that an increase in the thermal pressure at the base of the jet is also driven by the reconnection, which induces a train of slow-mode shocks propagating upward resulting in plasma upflows. Their findings contribute to the formation of jets and small-scale flows in the quiet-Sun corona where MMFs undergo into the low atmospheric reconnection.

Two outstanding issues, however, remain unsettled: i) what are the drivers of these winds in the outer corona? and ii) what are the source regions and what drivers enable the mass supply to the lower solar atmosphere (chromosphere-TR, and inner corona)? There exist several studies advocating the role of Alfvén waves as a possible answer to the first question. The ion-cyclotron waves at kinetic scales are visualized as one of the possible candidates to provide momentum and heat the outer coronal winds both in theory and observations (e.g. Ofman and Davila, 1995; Tu and Marsch, 1997; Tu et al., 2005; Suzuki and Inutsuka, 2005; Dwivedi and Srivastava, 2006; Jian et al., 2009, and references cited therein). The second question is crucial at present. The consensus of solar-wind research, however, is unclear as to the origin of the mass supply to the supersonic wind. Tian et al. (2010) have found upflows in the open-field lines of coronal holes starting in the solar transition region and interpreted this as an evidence of the fast solar wind in the polar coronal holes. As far as the slow solar wind is concerned, the outflows at the boundaries of active regions can contribute at larger spatio-temporal scales to the mass supply as an expansion of the loops lying over these active regions (Harra et al., 2008). It has been shown recently that collimated jet eruptions can also contribute to the formation of the solar wind (Madjarska et al., 2013). The magnetic-field topology of structures such as jets (e.g. spray surges) may not contribute to the solar wind (Uddin et al., 2012). The contributions from the confined ejecta in the solarwind formation depend mainly on the local magnetic-field topology and plasma conditions.

The question remains on whether the mass supply to the slow solar wind comes from the lower atmosphere expanding along the curved coronal fields. The question is what are the potential physical drivers? It has been found that the open-field lines at the boundary of active regions reconnect periodically with closed-field lines to guide the plasma motion in the form of solar wind (Harra et al., 2008). Similar examples are reported at the boundary of the coronal holes at small spatial scales as the source of slow solar wind (Subramanian, Madjarska, and Doyle, 2010). Therefore, an alternative option may be magnetic reconnection 
as a potential mechanism in the formation of slow solar wind. Apart from magnetic reconnection, the wave-heating scenario can shed light on the slow solar-wind source regions. Schmidt and Ofman (2011) have found that the energy stored in the slow magnetoacoustic waves propagating toward the higher atmosphere within expanding loops. This may be a potential candidate for the acceleration and formation of the slow solar wind. Wave activity at the bases of the fast (polar coronal holes) and slow (equatorial corona) solar wind can be important to power the energized plasma at greater heights up to the corona where it can be triggered supersonically in interplanetary space (e.g. Harrison, Hood, and Pike, 2002; Dwivedi and Srivastava 2006, 2008; De Pontieu et al., 2007; McIntosh et al., 2011; McIntosh, 2012, and references cited therein). Thus, there seems to be compelling evidence for the role of magnetic reconnection and wave phenomena in the solar-wind source region.

In the present article, we report the evidence of the outflowing magnetic arches acting as coronal funnels at the eastern boundary of an AR 10940 loop system observed on 5 February 2007. These coronal funnels seem to open up in the higher atmosphere to transport the outflowing plasma. Their footpoints are rooted in the boundary of the active region. They are the most likely heated regions that result in the activation of the outflowing plasma. We present a 2D MHD simulation of the open and expanding-funnel-type model atmosphere in which a rarefied and hot region is implemented near the footpoint that exhibits plasma perturbations similar to the observations. In Section 2 we summarize the observational results. We present the numerical model in Section 3. Discussion and conclusions are given in the last section.

\section{Observational Results}

The active region AR 10940 was observed by a one-arcsecond-slit scan of EUV Imaging Spectrometer (EIS: Culhane et al., 2006) onboard the Hinode spacecraft on 5 February 2007. The EIS is an imaging spectrometer of which 40- and 266-arcsecond slots are used for the image analyses using the light curves and emission per pixel. The one- and twoarcsecond slits are utilized for spectral and Doppler analyses using spectral-line profiles. EIS observes in two modes: i) scan; ii) sit-n-stare. The EIS observes high-resolution spectra in two wavelength intervals: 170-211 $\AA$ and 246-292 A using, respectively, its ShortWavelength (SW) and Long-Wavelength (LW) CCDs. The spectral resolution of the EIS is $0.0223 \AA$ per pixel. The analyzed observations were taken on 5 February 2007 and the data-set contains spectra of various lines formed at chromospheric, transition region (TR), and coronal temperatures. The scanning observation started at 12:14:12 UT and ended at 13:31:21 UT on 5 February 2007. The scanning steps were without any offset in the region containing a coronal active region and its eastern boundary with open and expanding magnetic arches where we are interested in the present investigation. This provides us with an opportunity to understand the plasma activity along the open-field regions at the eastern boundary of the active region in between the diffused loop systems that reach up to a higher height in the corona (Figure 1). We refer to these structures as "coronal funnels". Such flow regions are heated at their base exhibiting outflows. To understand the approximate magnetic-field geometry associated with AR 10940 and its surrounding region, we perform a potential field source surface (PFSS) extrapolation. Fan lines over the Solar and Heliospheric Observatory (SOHO)/Michelson Doppler Imager (MDI) observations are shown in Figure 2. It is clear that the core loops of the active region are bipolar and connect the central opposite magnetic polarities (white loops shown by the black arrow). Another set of closed-field lines connect to the east-side weak negative polarity and the central positive 

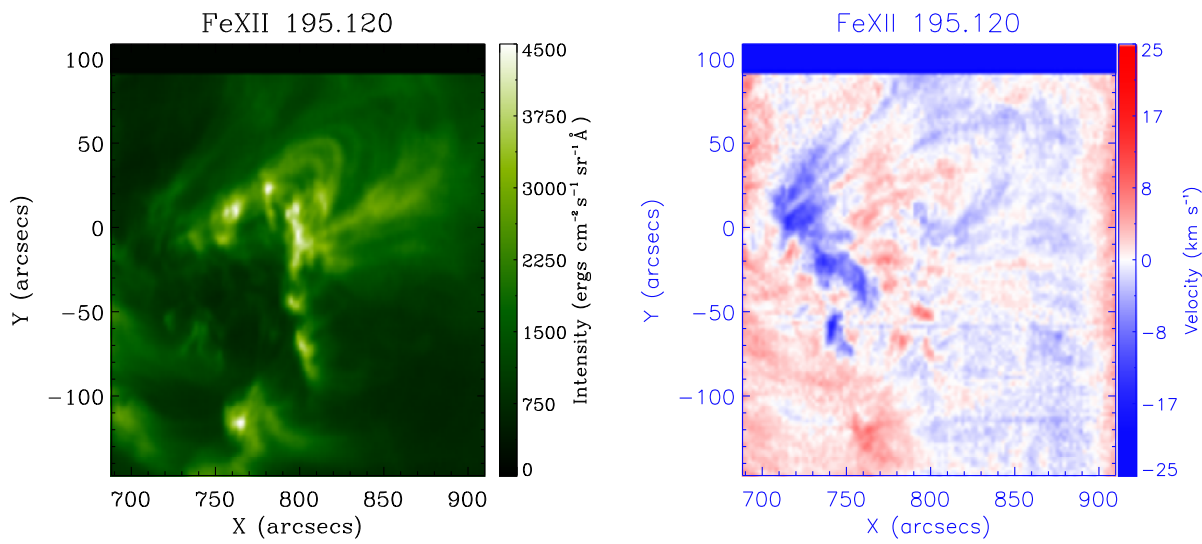

Figure 1 Intensity (left) and Doppler-velocity (right) maps of Active Region 10940 observed on 5 February 2007. The core loops and the open arches at its eastern boundary are clearly evident.

Figure 2 The magnetic-field polarities of AR 10940 and surrounding regions as observed by Solar and Heliospheric Observatory (SOHO)/Michelson Doppler Imager (MDI), and its potential field source surface extrapolation (PFSS). The black arrow indicates the core-loop system in the AR, while the south-east part of its boundary shows the large-scale open field lines extended into the upper corona. Some of these lines also connect to the coronal hole lying in the North of AR.

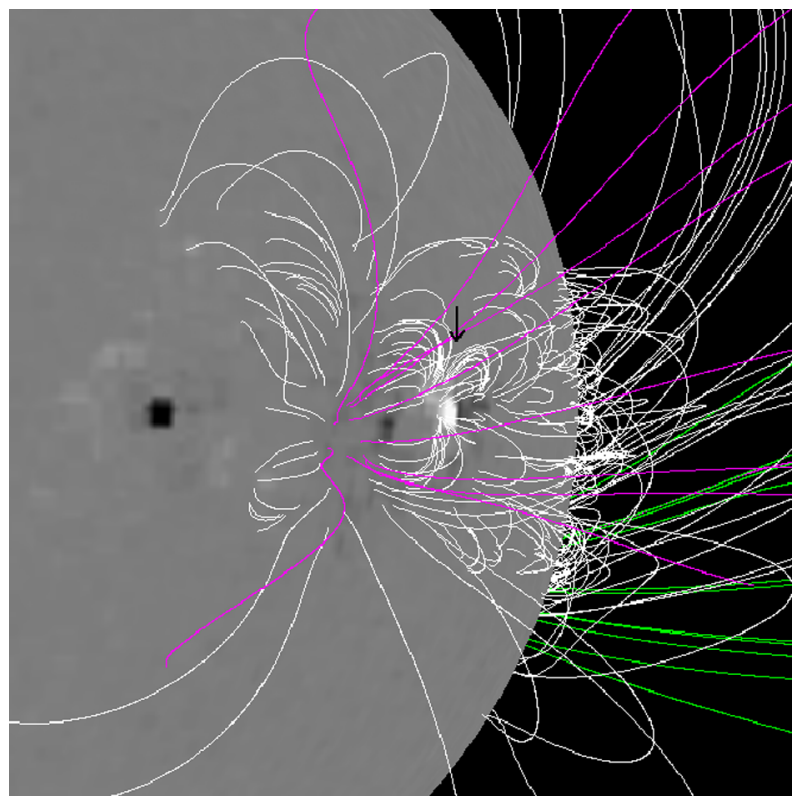

polarity. At the eastern boundary, there are weak patches of positive and negative polarities from where the open-field lines extend up to higher corona. These open-field lines form the large-scale coronal funnels at the eastern boundary of the active region. The lower parts of the loops at the eastern boundary can also serve as funnels up to a certain height in the corona. The white lines represent closed magnetic fields, while magenta and green lines represent the open fields that reach at the source surface, having opposite polarities. The funnels and the south-east boundaries of the AR are associated with the large-scale open field lines (magenta). They may also be the lower parts of the closed-loop system (white lines).

In order to obtain the velocity structures in such coronal funnels, we select the strongest EIS line [Fe XII 195.12 $\AA$ ] in our study. We aim for the understanding of 
the impulsively generated plasma outflows in such funnels and associated physical processes. The slit started the scanning of the polar coronal hole with $\left(X_{\text {cen }}, Y_{\text {cen }}\right) \approx$ (799.087 arcsecond, -19.185333 arcsecond). The observational windows acquired on the CCDs are 128 pixels high along the slit, and 111 pixels wide in the horizontal direction where spectra have a spectral resolution of $0.0222 \AA$ per pixel.

We apply the standard EIS data-reduction procedures and calibration files/routines to the data obtained from the EUV-telescope, which is the raw (zeroth-level) data. The subroutines are found in the sswidl software tree under the IDL environment (www.darts.isas.jaxa.jp/pub/ solar/ssw/hinode/eis/). These standard subroutines reduce the dark-current subtraction, cosmic-ray removal, flat-field correction, hot pixels, warm pixels, and bad/missing pixels. The data are saved in the level- 1 data file, and the associated errors are saved in the error file. We choose the clean and strong line Fe XII 195.12 A to examine the spatial variations of the intensity and Doppler velocity in the observations scanned over AR 10940 on 5 February 2007. We co-align the Fe XII 195.12 A map with respect to the long-wavelength CCD observations of He II $256.86 \AA$ by considering it as a reference image and by estimating its offset. The orbital and slit-tilt are also corrected in the data using the standard method described in the EIS-software notes. We perform a double Gaussian fit for the removal of the weak blending of Fe XII 195.18 $\AA$ line as per the procedure described by Young et al. (2009), which is also outlined in the EIS Software Note 17 . We constrain the weakly blended line 195.18 $\AA$ to have the same width as the line at $195.12 \AA$ and to fix an offset of $+0.06 \AA$ relative to it. It is to be noted that we perform the fitting on $2 \times 2$ pixel $^{2}$ binned data to enhance the signal-to-noise ratio and also to be obtain a reasonable fit.

Figure 1 displays the intensity (left) and Doppler-velocity (right) maps of the observed AR and open arches at its eastern boundary. The intensity map shows that the AR is made up of the core diffused loop systems lying at the lower heights in the plane perpendicular to the line-of-sight. In actuality, however, they may be tilted. These low-lying core loops exhibit high emission and downflows. At the eastern boundary, we have identified the open and expanding arches that exhibit coronal-funnel behavior to transport mass and energy into the higher corona. These expanding funnels may be the part of more quiescent and largescale loop systems opening higher up in the corona. It is seen in the enlarged Doppler map (see Figure 3) that four such regions are at least identified as blue-shifted and outflowing regions. As already noted, these could be the legs of higher and large-scale loops, which form the building blocks of the coronal funnels at relatively lower heights of the corona. They exhibit the slow and subsonic plasma outflows mostly spreading along their field lines with a maximum speed of about $10-15 \mathrm{~km} \mathrm{~s}^{-1}$.

Figure 3 shows the selection of path $1-4$ (top panel). The first two paths are drawn over the large-scale open and magnetic-field arches (lines) extending towards $\mathrm{QS}-\mathrm{CH}$ region in the North-East of this AR (not shown here). These two regions serve as the open and expanding funnel regions. Paths 3 and 4 are drawn to the expanding and blue-shifted regions that can also serve as coronal funnels. However, they are actually the lower parts of some core-loop systems. On this account, we have selected two coronal funnels (1 and 2), while the others ( 3 and 4) serve as funnels but are probably associated at larger heights with the curved-loop magnetic field. The middle and bottom panels (left to right), respectively, show the variation of projected L.O.S. Doppler velocity along paths $1-4$ with height in these funnels. For funnels 1 and 2, the footpoints exhibit stronger outflows with a maximum speed of $15 \mathrm{~km} \mathrm{~s}^{-1}$. At heights beyond $10 \mathrm{Mm}$, the outflow speed weakens in these funnels. This signifies the start of the outflows due to heating near the footpoints of these funnels; the outflows weaken with the height as we move away from the heating source. The other two funnels ( 3 and 4 ) are likely the legs of the diffused core loops, exhibiting the outflows at 

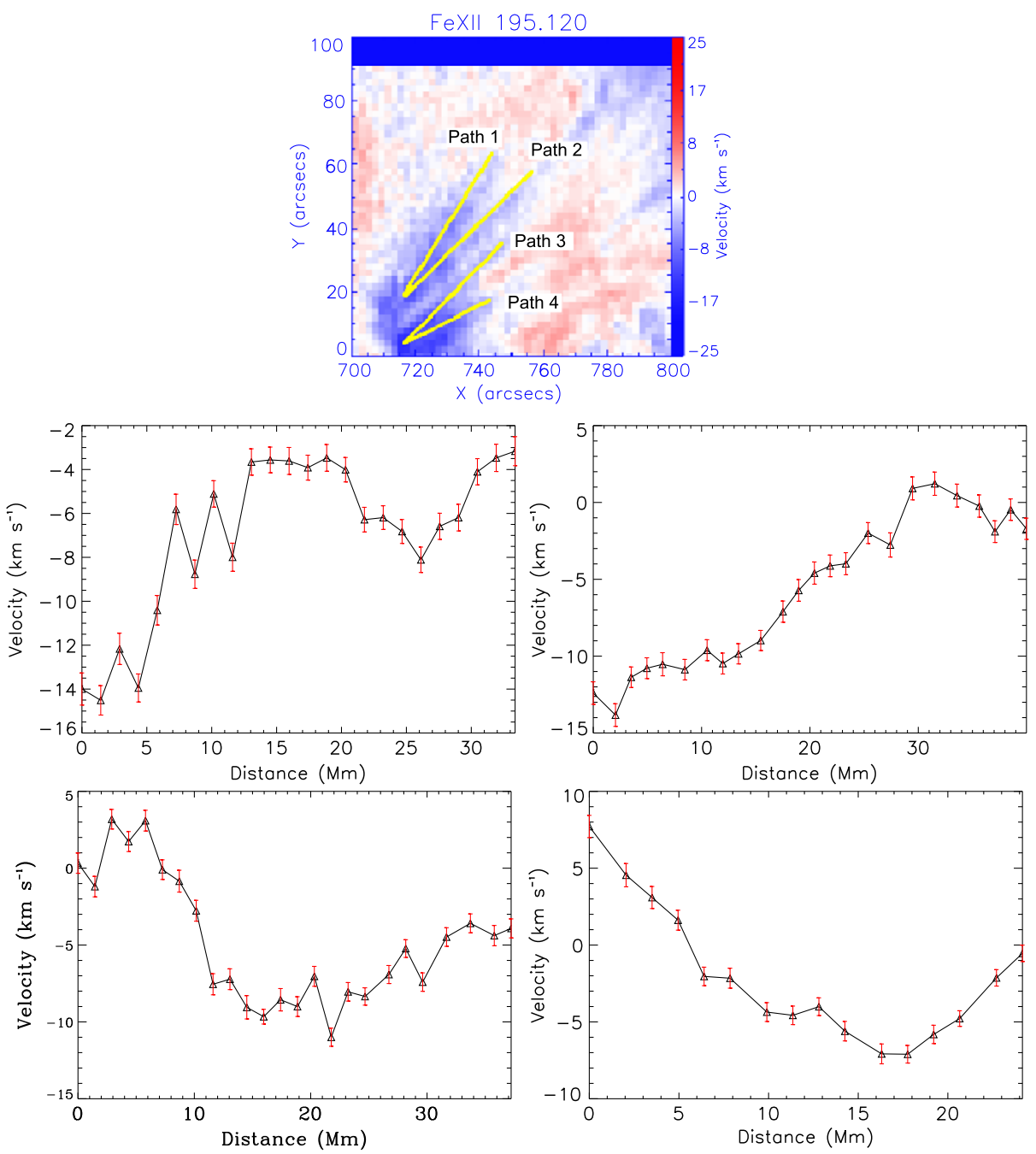

Figure 3 Top: the enlarged velocity map of the eastern boundary of AR 10940 showing the open and expanding funnels in which the plasma is outflowing. Middle and bottom: the variation of L.O.S. Doppler velocities along funnel 1 (middle-left), 2 (middle-right), 3 (bottom-left), and 4 (bottom-right).

certain lower heights with a maximum speed of $10 \mathrm{~km} \mathrm{~s}^{-1}$. This indicates different locations of the heating. It is also noted that the outflows diminish at lower heights compared to the corresponding ones in funnels 1 and 2 in the form of open arches. This is because of their association with the curved loops at greater heights where plasma is trapped and flows downward in order to maintain a new equilibrium. It is also noted that the flow structure is more gentle in the open funnels 1 and 2. However, it is greater at the footpoint and decreases with height. In funnels 3 and 4, which are the lower parts of curved loops, the generation of the flow is rather impulsive. The outflow starts at a certain height above the loop's footpoint. It increases up to a certain distance and decreases thereafter. This shows that impulsive heating is at work near the loop footpoint, which causes enhanced upflows up to a certain distance. The downflowing plasma from the upper part of the loop may counteract with the 
upflows. The red-shifted apex of the core-loop system is clearly evident in Figures 1 and 3. Funnels 3 and 4 are the lower parts of this loop system.

In the next section, we outline the details of the $2 \mathrm{D}$ numerical simulation of such observed expanding coronal funnels and their plasma dynamics. We solve a set of ideal MHD equations in the appropriate VAL-III C initial temperature conditions and model atmosphere using the FLASH code.

\section{Numerical Model of the Hot Plasma Outflows in Coronal Funnels}

\subsection{Model Equations}

Our model system starts from a gravitationally stratified solar atmosphere, which can be described by the ideal two-dimensional (2D) MHD equations:

$$
\begin{aligned}
\frac{\partial \varrho}{\partial t}+\nabla \cdot(\varrho \boldsymbol{V}) & =0, \\
\varrho \frac{\partial \boldsymbol{V}}{\partial t}+\varrho(\boldsymbol{V} \cdot \nabla) \boldsymbol{V} & =-\nabla p+\frac{1}{\mu}(\nabla \times \boldsymbol{B}) \times \boldsymbol{B}+\varrho \boldsymbol{g}, \\
\frac{\partial p}{\partial t}+\nabla \cdot(p \boldsymbol{V}) & =(1-\gamma) p \nabla \cdot \boldsymbol{V}, \\
\frac{\partial \boldsymbol{B}}{\partial t} & =\nabla \times(\boldsymbol{V} \times \boldsymbol{B}), \quad \nabla \cdot \boldsymbol{B}=0 .
\end{aligned}
$$

Here $\varrho, \boldsymbol{V}, \boldsymbol{B}, p=\frac{k_{\mathrm{B}}}{m} \varrho T, T, \gamma=5 / 3, \boldsymbol{g}=(0,0,-g)$ with its value $g=274 \mathrm{~m} \mathrm{~s}^{-2}, m$, and $k_{\mathrm{B}}$ are the mass density, flow velocity, magnetic field, gas pressure, temperature, adiabatic index, gravitational acceleration, mean particle mass, and Boltzmann's constant, respectively. It should be noted that we do not consider radiative cooling and thermal conduction in our present model for the sake of simplicity. We simulate only the dynamics of the plasma outflows to compare them with that of the observed coronal funnels.

\subsection{Initial Conditions}

We assume that the solar atmosphere is at rest $\left[\boldsymbol{V}_{\mathrm{e}}=\mathbf{0}\right]$ in equilibrium with a current-free magnetic field $\left[\nabla \times \boldsymbol{B}_{\mathrm{e}}=\mathbf{0}\right]$. As a result, the magnetic field is force-free:

$$
\left(\nabla \times \boldsymbol{B}_{\mathrm{e}}\right) \times \boldsymbol{B}_{\mathrm{e}}=\mathbf{0} .
$$

The divergence-free constraint is satisfied automatically if the magnetic field is specified by the magnetic-flux function $[A(x, y)]$ as

$$
\boldsymbol{B}_{\mathrm{e}}=\nabla \times(A \hat{\mathbf{z}}) .
$$

Here the subscript "e" corresponds to equilibrium quantities. We set a curved magnetic field by choosing

$$
A(x, y)=S \frac{x-a}{(x-a)^{2}+(y-b)^{2}},
$$

where $S$ is the strength of a magnetic pole and $(a, b)=(0,-10) \mathrm{Mm}$ is its position. For such a choice of $(a, b)$, the magnetic-field vectors are weakly curved to represent the expanding coronal funnels. 

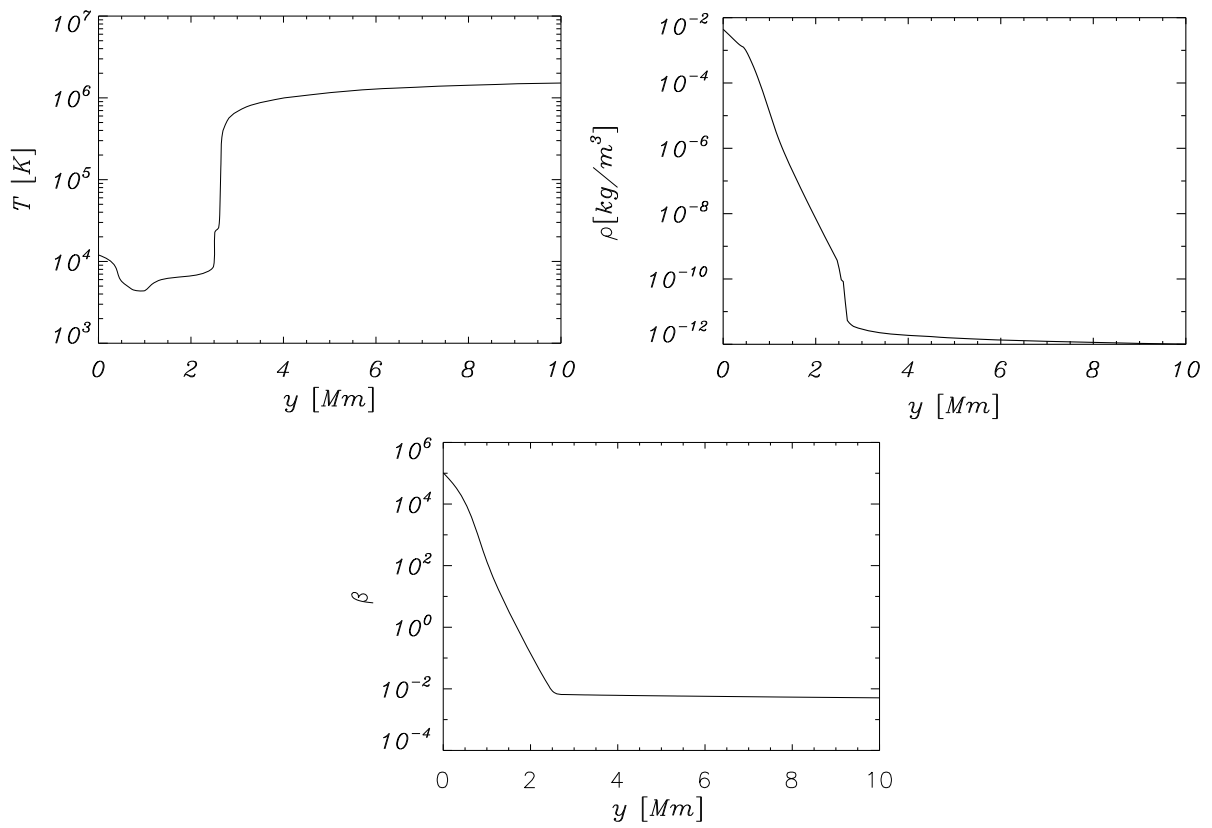

Figure 4 Equilibrium profiles of temperature (top-left panel), mass density (top-right panel), and plasma- $\beta$ (bottom panel) along the vertical line $[y]$ for $x=0$.

As a result of Equation (5) the pressure gradient is balanced by the gravity force:

$$
-\nabla p_{\mathrm{e}}+\varrho_{\mathrm{e}} \mathbf{g}=\mathbf{0} \text {. }
$$

With the ideal-gas law and the $y$-component of Equation (8), we arrive at

$$
p_{\mathrm{e}}(y)=p_{0} \exp \left[-\int_{y_{\mathrm{r}}}^{y} \frac{\mathrm{d} y^{\prime}}{\Lambda\left(y^{\prime}\right)}\right], \quad \varrho_{\mathrm{e}}(y)=\frac{p_{\mathrm{e}}(y)}{g \Lambda(y)},
$$

where

$$
\Lambda(y)=\frac{k_{\mathrm{B}} T_{\mathrm{e}}(y)}{m g}
$$

is the pressure scale height, and $p_{0}$ denotes the gas pressure at the reference level that we choose in the solar corona at $y_{\mathrm{r}}=10 \mathrm{Mm}$.

We take an equilibrium temperature profile $\left[T_{\mathrm{e}}(y)\right]$ (see top-left panel in Figure 4) for the solar atmosphere derived from the VAL-C atmospheric model of Vernazza, Avrett, and Loeser (1981) that is smoothly extended to the solar corona.

The transition region is located at $y \approx 2.7 \mathrm{Mm}$. There is an extended solar corona above the transition region, with the temperature minimum level located at $y \approx 0.9 \mathrm{Mm}$ below the solar chromosphere. Having specified $T_{\mathrm{e}}(y)$ (see Figure 4, left-top panel) with Equation (9), we obtain the corresponding gas-pressure and mass-density profiles.

\subsection{Numerical Scheme and Computational Grid}

Equations (1) - (4) are solved numerically using the FLASH code (Lee and Deane, 2009; Lee, 2013). This code uses a second-order, unsplit Godunov solver (Godunov, 1959) with 
Figure 5 Numerical blocks with their boundaries (solid lines).

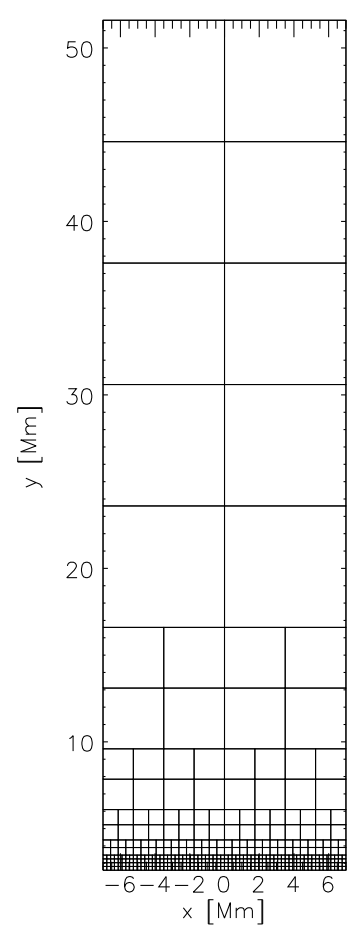

various slope limiters and Riemann solvers, as well as Adaptive Mesh Refinement (AMR) (Murawski and Lee 2011, 2012). We set the simulation box of $(-7,7) \mathrm{Mm} \times(2.6,51.6) \mathrm{Mm}$ along the $x$ - and $y$-directions (Figure 5). The lower boundary, where we apply the heating pulse, is set at $x_{0}=0 \mathrm{Mm}, y_{0}=2.6 \mathrm{Mm}$. We set and hold fixed all of the plasma quantities at all boundaries of the simulation region to their equilibrium values, which are given by Equations (6) and (9). As the magnetic field is curved and plasma is stratified gravitationally, open boundaries would not be a perfect choice with regard to the fixed boundary conditions. We have verified this experimentally. As the FLASH code uses a third-order accurate Godunov-type method, a characteristic method is already built into this procedure. However, the Riemann problem at the boundaries corresponds to open boundaries, resulting in numerically induced reflections from these boundaries. On the other hand, the fixed boundaries lead to negligibly small numerical reflections. Therefore, we adopt this approach in the numerical simulations. In addition, we modify the equilibrium mass density and gas pressure at the bottom boundary as

$$
\begin{aligned}
& \varrho(x, y, t)=\varrho_{\mathrm{e}}(y)\left[1+A_{\varrho} f(x, y) g(t)\right], \\
& p(x, y, t)=p_{\mathrm{e}}(y)\left[1+A_{\mathrm{p}} f(x, y) g(t)\right],
\end{aligned}
$$

where

$$
f(x, y)=\exp \left[-\frac{\left(x-x_{0}\right)^{2}}{w_{\mathrm{x}}^{2}}-\frac{\left(y-y_{0}\right)^{2}}{w_{\mathrm{y}}^{2}}\right]
$$

and

$$
g(t)=-\left[\exp \left(-\frac{t}{\tau}\right)-1\right]
$$


Here $A_{\varrho}$ and $A_{\mathrm{p}}$ are the amplitudes of the perturbations, $\left(x_{0}, y_{0}\right)$ are their initial position, and $w_{\mathrm{x}}, w_{\mathrm{y}}$ denote its widths along the $x$ - and $y$-directions, respectively. The symbol $\tau$ denotes the growth time of these perturbations. We set and hold fixed $A_{\varrho}=7, A_{\mathrm{p}}=2, x_{0}=0 \mathrm{Mm}$, $y_{0}=2.6 \mathrm{Mm}, w_{\mathrm{x}}=3 \mathrm{Mm}, w_{\mathrm{y}}=0.5 \mathrm{Mm}$, and $\tau=10$ seconds. These fixed boundary conditions perform much better than transparent boundaries, leading to only negligibly small numerical reflections of the wave signals from these boundaries.

In our modeling, we use an AMR grid with a minimum (maximum) level of refinement set to 3 (8) (see Figure 5). The refinement strategy is based on controlling numerical errors in mass density, which results in an excellent resolution of steep spatial profiles and greatly reduces numerical diffusion at these locations.

A standard procedure to check the magnitude of a numerically induced flow is to run the code for the equilibrium alone, without implementing any perturbation. We have verified that the numerically induced flow is of the order of $2 \mathrm{~km} \mathrm{~s}^{-1}$ in the solar corona, and the transition region is not affected by the spatial resolution which is about $20 \mathrm{~km}$ around the transition region. This resolution is much smaller than the width of the transition region $(\approx 200 \mathrm{~km})$ and the pressure scale height near the bottom, which is about $0.5-1 \mathrm{Mm}$. We have also performed grid-convergence studies by increasing the spatial resolution by a factor of two at the transition region. As the numerical results have been found essentially similar results for the finer and coarser grid, we have limited our analysis to the latter.

\subsection{Results of the Numerical Simulation and Comparison with Observations}

The results of the numerical simulations and their comparison with the observed plasma outflows are summarized as follows:

Figure 6 (first column) displays the velocity vectors plotted over the total velocity maps for $t=50,100,150,200,250,300,350,400,450$ seconds. The diverging magneticfield lines of the model funnel is also over-plotted in these snapshots. It is clear from the $t=50$ seconds snapshot that the implemented heating at the footpoint, just below the transition region, results in the alteration of the ambient plasma pressure, and the plasma starts flowing upward with a typical average speed of $40 \mathrm{~km} \mathrm{~s}^{-1}$ near the footpoint at this time. The outflow velocity is maximum near the heated region at the footpoint of the model funnel. This is higher than the observed line-of-sight outflow velocities $\left(10-16 \mathrm{~km} \mathrm{~s}^{-1}\right)$ in various coronal funnels above their footpoints (see Figure 3). The simulated outflow velocities greatly depend on the initial conditions of the model funnel and magnitude of the heating pulse. The observed trend of the flows in funnels 1 and 2 (see Figure 3) and the same derived from the model match each other (see Figures 6 and 7). At higher altitudes, above the heating location in these funnels, the outflow weakens which qualitatively matches with the results obtained from our model (see Figures 6 and 7). Our model fits better the plasmaflow conditions in the open coronal funnels (e.g. funnels 1 and 2) where the gentle flows start and expand due to their footpoint heating. The flow becomes steady at each height after 300 seconds in the model funnel when the heated plasma reaches the height of $10 \mathrm{Mm}$. This also indicates that the heating pulse is at work for a certain duration, and after some time the generated flows reach a new equilibrium. The physical scenario is in agreement with the open coronal funnels 1 and 2 (see Figures 3 and 7). Comparison of these two scenarios supports the outflow of the plasma due to heating. The heating causes thermal flows guided in the magnetic-field lines of the open coronal funnels, while it subsides away from the source. The physical behavior of the velocity field and its spatial distribution as observed by Hinode/EIS along each funnel are consistent with the velocity field at a particular temporal span of the numerical simulation when the outflowing plasma rises to a maximum height of $\approx 10 \mathrm{Mm}$. 

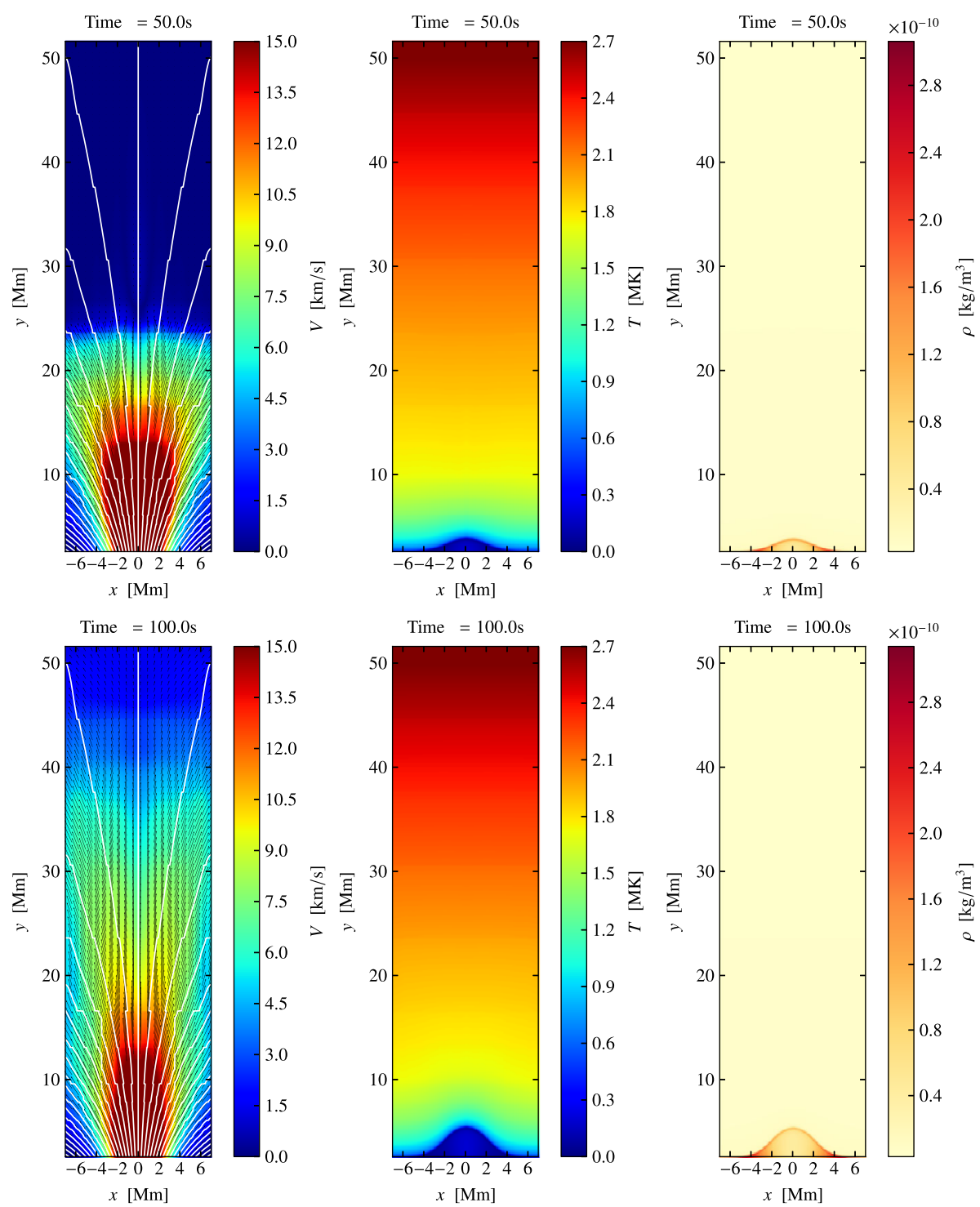

Figure 6 Numerical results (top to bottom rows): the velocity vectors superposed over the total velocity (left); the temperature (middle), and density (right) maps, for $t=50,100,200,300$ seconds. White lines represent the diverging magnetic-field lines. The complete movies are given as an electronic supplementary material for these numerical results.

For the funnels that are the lower parts of the curved-loop system (funnels 3 and 4; Figure 3), the energy release might occur where the blue-shift is enhanced at a certain height above the footpoint impulsively. Therefore, the locations in those observed funnels are identical to the energy-release site of the modeled funnel where outflows start as a result of heating. On the contrary, it seems that impulsive heating in funnels 3 and 4 causes the increment in the outflows up to a certain height and thereafter is balanced by the downflowing and trapped plasma from the loop apex. 


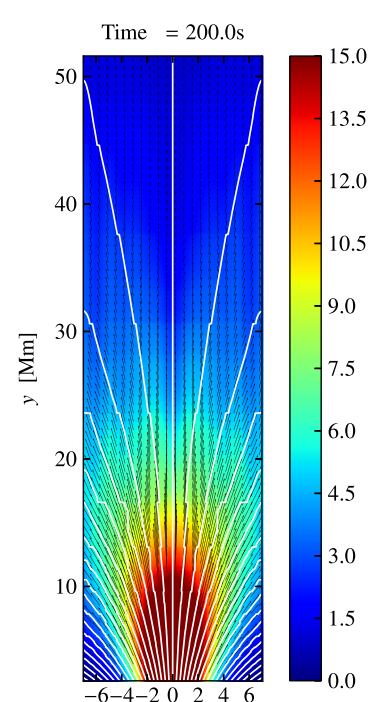

$x[\mathrm{Mm}]$

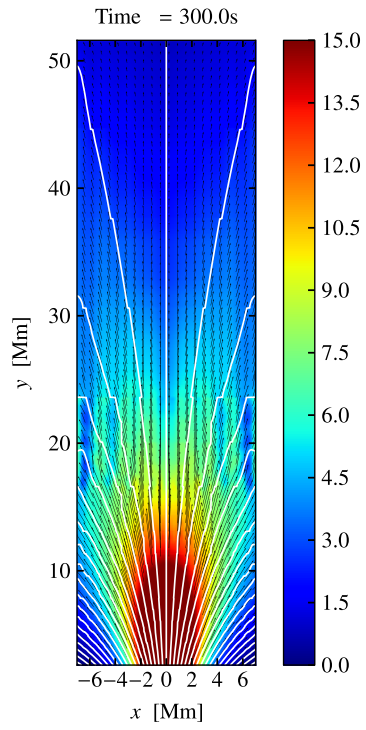

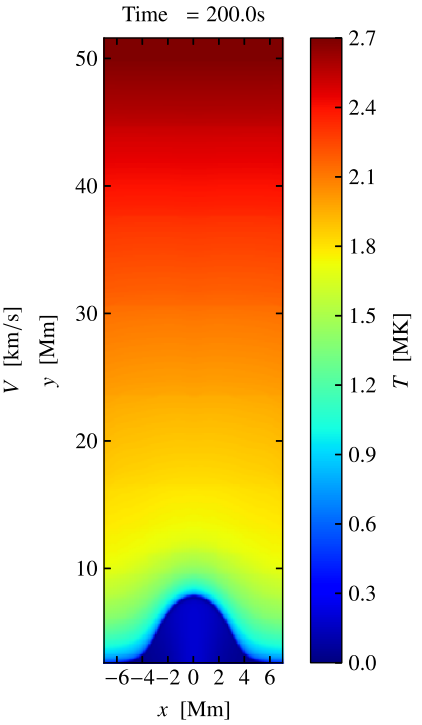

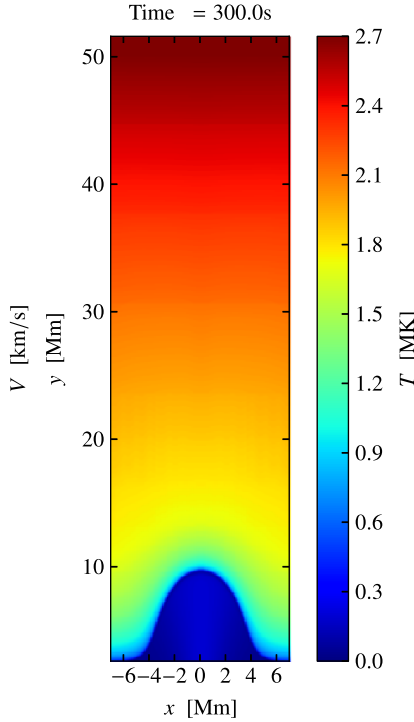

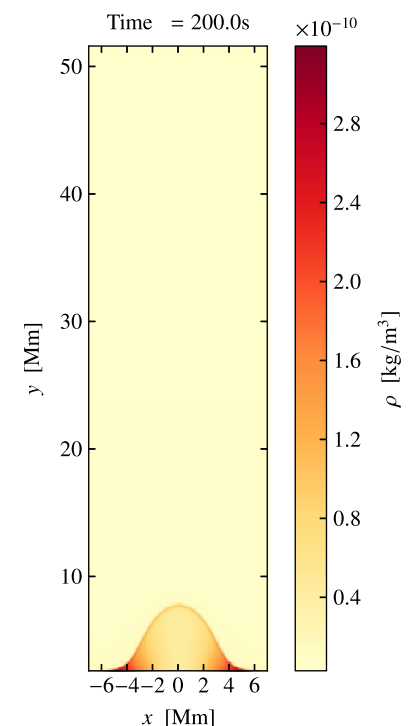

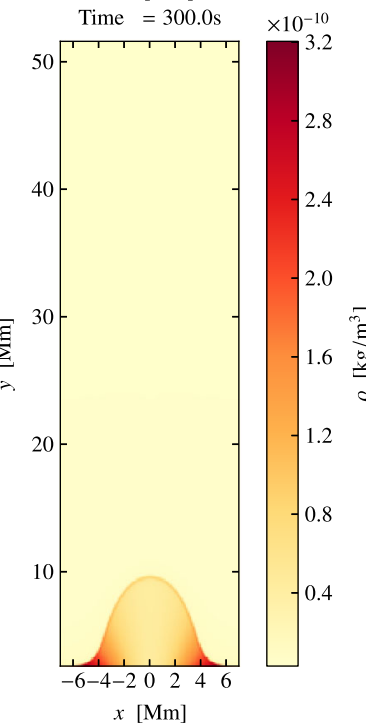

Figure 6 (Continued.)

Figure 6, second and third columns, respectively, display the temperature and density maps for $t=50,100,200$, and 300 seconds. It is clear that during the heating, the plasma maintained at inner coronal/TR temperatures (sub-MK and 1.0 MK; the hot plasma envelops the cool one) and with somewhat higher density, starts flowing from the footpoint of the model funnel toward greater heights. The plasma is denser near the footpoint. It flows along the open funnels toward higher heights. Thermal perturbations created the slow and subsonic flows of the plasma; therefore, it reaches up to only the lower heights. This is a situation similar to that of the transition region within the funnel being pushed upward due to the evolution of the thermal perturbation underneath. Sub-mega-Kelvin and denser plasma from 

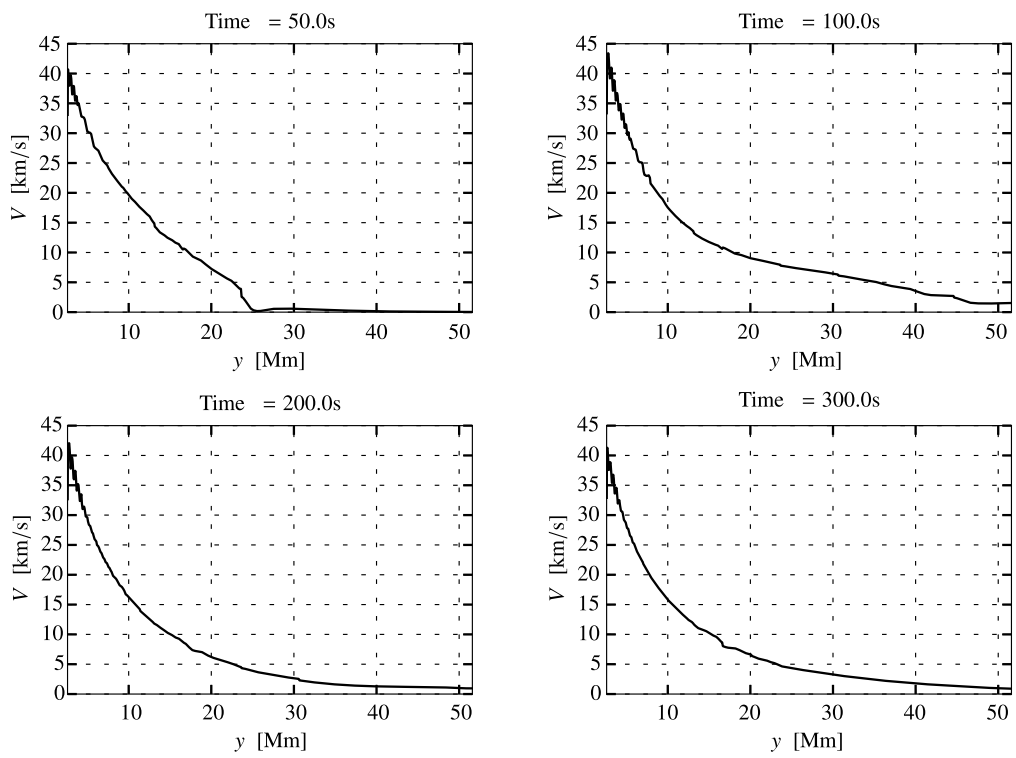

Figure 7 Velocity profiles plotted versus the height along the model funnel at 50, 100, 200, 300 seconds.

lower solar atmosphere move up and is enveloped by the hot coronal plasma. The denser plasma maintained at the TR and inner coronal temperatures is visible up to a height of $10 \mathrm{Mm}$ in the model funnel. This is consistent with the observations. Only the lower parts of the coronal arches are associated with enhanced fluxes and thus with higher densities, while their higher parts are less intense and denser regions (see Figure 1, left panel).

\section{Discussion and Conclusions}

We have presented observations of outflowing coronal arches (coronal funnels 1 and 2) lying at the eastern boundary of the AR 10940 observed on 5 February 2007, 12:1513:31 UT. The scanning observations show that these arches open up in the nearby quietSun corona and exhibit plasma outflows maintained at coronal/TR temperature of around 1.0 MK. They serve as the expanding coronal funnels from which the plasma moves to the higher corona and serves as a source of the slow solar wind (Harra et al., 2008). The plasma outflows may be generated in such open-field regions because of the low atmospheric reconnections between the open- and closed-field lines (Subramanian, Madjarska, and Doyle, 2010). Episodic heating mechanisms are now well observed and interpreted as the drivers of outflowing plasma in the curved coronal loops (Klimchuk, 2006; Del Zanna, 2008; Brooks and Warren, 2009). The steady heating may generate the hot-plasma upflows in the corona (Tripathi et al., 2012).

There has so far been little effort made to model the plasma outflows in the corona and that too in an entirely different context of the large-scale evolution of coronal magnetic field. Murray et al. (2010) have modeled in 3D the origin and driver of coronal outflows, and found that outflows are the result of the expansion of an active region during its development. Harra et al. (2012) have modeled the AR coronal outflows as a consequence of compression during the creation and annihilation of the magnetic-field lines. 
However, our objective of the present investigation is to implement and test our 2D numerical-simulation model of the localized expanding coronal funnels with Hinode/EIS observations of the outflowing open field arches (i.e. funnels) at the boundary of AR 10940. We solve the set of ideal MHD equations in the appropriate VAL-III C initial temperature conditions and model atmosphere using the FLASH code. The key ingredient of our model is the implementation of realistic ambient solar atmosphere, e.g. realistic temperature, presence of the TR, implementation of expanding coronal fields, stratified atmosphere in the initial equilibrium, which significantly affect such plasma dynamics. We have implemented a rarefied and hotter region at the footpoint of the model funnel that triggers the evolution of the slow and subsonic plasma perturbations propagating outward in the form of plasma flows similar to the observed dynamics. We implemented the localized heating below the transition region at the footpoint of the funnel. The plasma is considered rarefied in the horizontal direction, mimicking the structured, open, and expanding magnetic funnels. The heated plasma evolves and exhibits the plasma perturbations as has been observed as outlined in the Hinode/EIS observations. The outflows start at the base of the funnel that further weakens with the height, which is suggestive of the plasma dynamics due to heating near the footpoint. A similar physical scenario is observed in the selected outflowing magnetic arches mimicking the coronal funnels 1 and 2 at the eastern boundary of AR 10940.

We conclude that the implemented episodic heating can excite plasma outflows in expanding coronal funnels residing at boundary of solar active regions as well as in the quietSun. These slow, subsonic plasma outflows may not be launched at higher altitudes in the corona. We have examined the presence of hot and denser plasma up to $10 \mathrm{Mm}$ height as triggered by thermal perturbations in our model. Observations also show that the plasma only rises significantly in the lower parts of these funnels up to inner coronal heights with significant intensity and velocity distributions. However, even if such flows reach up to the inner coronal heights of $10 \mathrm{Mm}$ in these funnels, they may also contribute to the mass supply to the slow solar wind. Therefore, our model and observations invoke the dynamics of the plasma in the localized coronal funnels, which may be important candidates to transport mass and energy into the inner corona. However, more observational studies should be performed with new spectroscopic data (e.g. Interface Region Imaging Spectrograph (IRIS)) to compare with our proposed 2D model, specifically under the physical conditions of different types of localized flux tubes in the solar atmosphere, which can serve as plasma outflowing regions due to episodic heating.

Acknowledgements We thank the referees for their valuable suggestions, which improved the manuscript considerably. We acknowledge the use of Hinode/EIS observations. AKS acknowledges the support of the International Exchange Scheme (Royal Society) between India and UK. The software used in this work was in part developed by the DOE-supported ASCI/Alliance Center for Astrophysical Thermonuclear Flashes at the University of Chicago.

Open Access This article is distributed under the terms of the Creative Commons Attribution License which permits any use, distribution, and reproduction in any medium, provided the original author(s) and the source are credited.

\section{References}

Brooks, D.H., Warren, H.P.: 2009, Astrophys. J. Lett. 703, L10. DOI.

Culhane, J.L., Doschek, G.A., Watanabe, T., Smith, A., Brown, C., Hara, H., et al.: 2006 In: SPIE 6266. DOI.

De Pontieu, B., McIntosh, S.W., Carlsson, M., Hansteen, V.H., Tarbell, T.D., Schrijver, C.J., Title, A.M., et al.: 2007, Science 318, 1574. DOI.

Del Zanna, G.: 2008, Astron. Astrophys. 481, L69. DOI. 
Dwivedi, B.N., Srivastava, A.K.: 2006, Solar Phys. 237, 143. DOI.

Dwivedi, B.N., Srivastava, A.K.: 2008, New Astron. 13, 581. DOI.

Godunov, S.K.: 1959, Sb. Math. 47, 271.

Habbal, S.R., Woo, R., Fineschi, S., O’Neal, R., Kohl, J., Noci, G., Korendyke, C.: 1997, Astrophys. J. Lett. 489, L103. DOI.

Harra, L.K., Sakao, T., Mandrini, C.H., Hara, H., Imada, S., Young, P.R., van Driel-Gesztelyi, L., Baker, D.: 2008, Astrophys. J. Lett. 676, L147. DOI.

Harra, L.K., Archontis, V., Pedram, E., Hood, A.W., Shelton, D.L., van Driel-Gesztelyi, L.: 2012, Solar Phys. 278, 47. DOI.

Harrison, R.A., Hood, A.W., Pike, C.D.: 2002, Astron. Astrophys. 392, 319.

Hassler, D.M., Dammasch, I.E., Lemaire, P., Brekke, P., Curdt, W., Mason, H.E., Vial, J.-C., Wilhelm, K.: 1999, Science 283, 810. DOI.

Jian, L.K., Russell, C.T., Luhmann, J.G., Strangeway, R.J., Leisner, J.S., Galvin, A.B.: 2009, Astrophys. J. 701, L105. DOI.

Klimchuk, J.A.: 2006, Solar Phys. 234, 41. DOI.

Lee, D.: 2013, J. Comput. Phys. 243, 269. DOI.

Lee, D., Deane, A.E.: 2009, J. Comput. Phys. 228, 952. DOI.

Madjarska, M., Huang, Z., Subramanian, S., Doyle, J.G.: 2013 In: EGUGA 15, 2455.

McIntosh, S.W.: 2012, Space Sci. Rev. 172, 69.

McIntosh, S.W., de Pontieu, B., Carlsson, M., Hansteen, V., Boerner, P., Goossens, M.: 2011, Nature 475, 477.

Murawski, K., Lee, D.: 2011, Bull. Pol. Acad. Sci., Tech. Sci. 59, 81. DOI.

Murawski, K., Lee, D.: 2012, Control Cybern. 42, 35.

Murray, M.J., Baker, D., van Driel-Gesztelyi, L., Sun, J.: 2010, Solar Phys. 261, 253. DOI.

Ofman, L., Davila, J.M.: 1995, J. Geophys. Res. 100, 23413. DOI.

Sakao, T., Kano, R., Narukage, N.: 2007, Science 318, 1585. DOI.

Schmidt, J.M., Ofman, L.: 2011, Astrophys. J. 739, 75. DOI.

Slemzin, V., Harra, L., Urnov, A., Kuzin, S., Goryaev, F., Berghmans, D.: 2013, Solar Phys. 286, 157. DOI.

Subramanian, S., Madjarska, M.S., Doyle, J.G.: 2010, Astron. Astrophys. 516, A50. DOI.

Suzuki, T.K., Inutsuka, S.-I.: 2005, Astrophys. J. Lett. 632, L49. DOI.

Tian, H., Tu, C.-Y., Marsch, E., He, J., Kamio, S.: 2010, Astrophys. J. Lett. 709, L88. DOI.

Tripathi, D., Mason, H.E., Del Zanna, G., Bradshaw, S.: 2012, Astrophys. J. Lett. 754, L4. DOI.

Tu, C.-Y., Marsch, E.: 1997, Solar Phys. 171, 363. DOI.

Tu, C.-Y., Marsch, E.: 2001, J. Geophys. Res. 106, 8233. DOI.

Tu, C.-Y., Zhou, C., Marsch, E., Xia, L.-D., Zhao, L., Wang, J.-X., Wilhelm, K.: 2005, Science 308, 519. DOI.

Uddin, W., Schmieder, B., Chandra, R., Srivastava, A.K., Kumar, P., Bisht, S.: 2012, Astrophys. J. $752,70$. DOI.

Vernazza, J.E., Avrett, E.H., Loeser, R.: 1981, Astrophys. J. 45, 635. DOI.

Wilhelm, K., Dammasch, I.E., Marsch, E., Hassler, D.M.: 2000, Astron. Astrophys. 353, 749.

Yang, L., He, J., Hardi, P., Tu, C., Zhang, L., Feng, X., Zhang, S.: 2013, Astrophys. J. 777, 16. DOI.

Young, P.R., Watanabe, T., Hara, H., Mariska, J.T.: 2009, Astron. Astrophys. 495, 587. DOI. 